\title{
OMEGA-3 FATTY ACIDS AND VITAMIN D IN IMMOBILISATION: PART A- MODULATION OF APPENDICULAR MASS CONTENT, COMPOSITION AND STRUCTURE
}

\author{
E.L. BOSTOCK, C.I. MORSE, K. WINWOOD, I.M. MCEWAN, G.L. ONAMBÉLÉ
}

\begin{abstract}
Health, Exercise and Active Living Research Centre, Department of Exercise \& Sport Science, Manchester Metropolitan University, Crewe Green Road, Crewe, CW1 5DU, United Kingdom. Corresponding author: Gladys Onambele-Pearson, Health, Exercise and Active Living Research Centre, Department of Exercise \& Sport Science, Manchester Metropolitan University, Crewe Green Road, Crewe, CW1 5DU, United Kingdom. Tel: +44 (0) 161247 5594; Email: g.pearson@mmu.ac.uk
\end{abstract}

\begin{abstract}
Objectives: Muscle size decreases in response to short-term limb immobilisation. This study set out to determine whether two potential protein-sparing modulators (eicosapentaenoic acid and vitamin D) would attenuate immobilisation-induced changes in muscle characteristics. Design: The study used a randomised, double-blind, placebo-controlled design. Setting: The study took part in a laboratory setting. Participants:

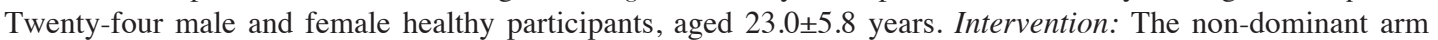
was immobilised in a sling for a period of nine waking hours a day over two continuous weeks. Participants were randomly assigned to one of three groups: placebo ( $\mathrm{n}=8$, Lecithin, $2400 \mathrm{mg}$ daily), omega-3 ( $\omega-3)$ fatty acids $(\mathrm{n}=8$, eicosapentaenoic acid (EPA); $1770 \mathrm{mg}$, and docosahexaenoic acid (DHA); $390 \mathrm{mg}$, daily) or vitamin D ( $n=8,1,000$ IU daily). Measurements: Muscle and sub-cutaneous adipose thickness (B-mode ultrasonography), body composition (DXA) and arm girth (anthropometry) were measured before immobilisation, immediately on removal of the sling and two weeks after re-mobilisation. Results: Muscle thickness (-5.4 $\pm 4.3 \%)$, upper and lower arm girth $(-1.3 \pm 0.4$ and $-0.8 \pm 0.8 \%$, respectively), lean mass $(-3.6 \pm 3.7 \%)$ and bone mineral content (BMC) $(-2.3 \pm 1.5 \%)$ decreased significantly with limb immobilisation in the placebo group $(\mathrm{P}<0.05)$. Despite no significant effect of group, $\omega-3$ and vitamin $D$ supplementation showed trends $(\mathrm{p}>0.05)$ towards attenuating the decreases in muscle thickness, upper/lower arm girths and BMC observed in the placebo group. The $\omega-3$ supplementation group demonstrated a non-significant attenuation of the decrease in DXA quantified lean mass observed in the placebo group. Sub-cutaneous adipose thickness increased in the placebo group $(\mathrm{P}<0.05)$. $\omega-3$ and vitamin $\mathrm{D}$ both blunted this response, with $\omega-3$ having a greater effect $(\mathrm{P}<0.05)$. All parameters had returned to baseline values at the re-mobilisation phase of the study. Conclusion: Overall, at the current doses, $\omega-3$ and vitamin D supplementation only attenuated one of the changes associated with non-injurious limb immobilisation. These findings would necessitate further research into either a) supplementation linked to injuryinduced immobilisation, or b) larger doses of these supplements to confirm/refute the physiological reserve potential of the two supplements.
\end{abstract}

Key words: Docosahexaenoic acid, eicosapentaenoic acid, immobilisation, lecithin, omega 3, vitamin D.

\section{Introduction}

Skeletal muscle undergoes structural adaptations in response to changes in functional demand. Prolonged periods of reduced muscle activity and mechanical loading, e.g. immobilisation, limb-suspension or bed-rest, result in changes in skeletal muscle structure and function, bone mineral density (BMD) and intermuscular adipose content (1-6). Musculoskeletal trauma and sports injuries are often treated with orthopaedic surgery/ limb immobilisation, which inevitably leads to periods of inactivity and disuse. The prognosis of orthopaedic surgery patients is poor especially in the older population with an $\sim 6$ to $17 \%$ mortality rate within three years of hip and knee joint replacement (7). Exercise could be beneficial in these circumstances, but is not always practical. In addition, there is a notable need to identify non-pharmacological interventions since polypharmacy in itself is conducive to skeletal tissue loss (8).

Eicosapentaenoic acid (EPA) is an n-3 polyunsaturated fatty acid with anti-inflammatory properties, which is synthesised from ingested alpha-linolenic acid or consumed in fish, or in fish oil, such as sardines and cod liver oil. Despite there being no established Dietary Reference Intake for n-3 fatty acids, adequate intake (AI) is set at 1.6 and $1.1 \mathrm{~g} /$ day for men and women, respectively (9). There is evidence to suggest that EPA may reduce the pro-inflammatory cytokines associated with muscle damage-induced inflammation (10). Magee et al. (2008) demonstrated in vitro that EPA inhibits the effects of TNF- $\alpha$ by reducing its apoptotic effects and enabling myogenesis (10). It is unclear whether this supplement would have a beneficial effect during immobilisation, where it is generally accepted that there is muscle atrophy (11), which is associated with decreased protein synthesis (12) but scant evidence for increased protein breakdown (13).

Another non-pharmacological agent that may potentially be used against the asthenia and sarcopenia induced through immobilisation is vitamin D. Vitamin D is required to absorb calcium and phosphorus and plays a crucial role in maintaining bone, muscle function, modulation of cell growth, neuromuscular and immune functions, and reduction of 
inflammation. The main source of vitamin D is sunlight, with smaller amounts found in certain foods. The Recommended Dietary Allowance for vitamin D is 600 IU/day (14). Vitamin D supplementation is another way of making sure the recommended allowance is achieved. Vitamin D has been shown to have direct effects on muscle (15); however, the exact mechanisms remain unknown. To date, research has indicated an association between genetic variation in the vitamin D receptor gene and muscle strength, fat mass and body mass in premenopausal women (16). Moreover, vitamin D has been reported to impact on both the trans-membranous flows of calcium and phosphate in skeletal muscle, and the synthesis rate of contractile properties (17). Vitamin D supplementation reduced falls by $49 \%$ and improved musculoskeletal function in frail elderly women with vitamin D deficiency (18). It remains to be seen whether vitamin D supplementation in healthy persons with no known vitamin D deficiency, would lead to any enhancement in muscle structural and contractile properties in the presence of immobilisation.

In the present study, an arm immobilisation model was chosen as it is relatively less restrictive on daily life and causes fewer burdens on participants. The aim was to determine the role that the two potential protein-sparing modulators (EPA or vitamin D supplementation) may play in attenuating atrophy induced through a model that would emulate relatively short-term decreased local mobility/activity in humans. The differential effect of immobilisation on in vivo muscle (thickness), limb composition (lean mass, bone parameters and fat mass), and anthropometry (arm girth) was systematically monitored. Participants received either omega $3(\omega-3$, a fish oil of a complex of EPA and docosahexaenoic acid (DHA)), vitamin D or placebo (Lecithin), hereafter simply referred to as EPA, vitamin D or placebo supplementation. It was hypothesised that muscle thickness, lean mass and arm girth will decrease with limb immobilisation. It was also hypothesised that EPA will be the most effective supplement at minimising these changes, since it is understood to act on the protein synthesis pathways.

\section{Method}

\section{Participants}

Twenty-four healthy volunteers were recruited from the local university campus. All participants provided written informed consent before taking part in this study, which was approved by the local Ethics Committee of Manchester Metropolitan University. Exclusion criteria were any conditions requiring the use of medication likely to affect muscle function or musculoskeletal health (e.g. statins and oral steroids), and any current or history of kidney/liver disease, as those suffering with such conditions are more susceptible to the side effects of nutritional supplementation. An in-house designed physical activity and general health questionnaire, generally used in our research laboratories for teaching and research purposes, was completed by each participant, to ascertain health, habitual physical exercise levels and supplement history prior to the study. This questionnaire confirmed all participants were recreationally active (defined here as undertaking three hours or less of low-to-moderate intensity exercise per week.), free from recent (last 6 months) upper limb injury, were not currently taking any supplements and had no history of $\omega-3$ or vitamin D supplementation within at least the last year. Participants were randomly assigned to one of three groups: placebo [PLA: $\mathrm{n}=8$ ]; EPA [EPA: $\mathrm{n}=8$ ]; or vitamin D [Vit-D: $\mathrm{n}=8$ ]. For anthropometric measurements (height, weight and BMI) in our three populations, please see the results section.

\section{Study design}

The study used a randomised, double-blind, placebocontrolled design. All participants attended a familiarisation session at least one week prior to the first testing session. All testing sessions were completed after an overnight fast. After baseline testing, the non-dominant arm was immobilised in a sling, with the correct sling wearing procedure demonstrated to each participant (Figure 1). Participants were required to wear the sling for nine waking hours a day for two continuous weeks, removal of the sling was permitted only when necessary (e.g. taking a bath/shower, driving, sleeping etc.), minimising any movement medio-laterally at the elbow and shoulder, whilst requiring participants to not contract the upper musculature (including the hands) during the hours of immobilisation. Measures of upper arm muscle and subcutaneous fat thickness, body composition (lean mass, bone parameters and fat mass), and upper and lower arm girth were taken immediately before immobilisation (Pre), on removal of the sling (Post), and two weeks after re-mobilisation (Post2).

The placebo group consumed two $1200 \mathrm{mg}$ capsules of Soya Lecithin (Holland \& Barrett, UK) a day, each daily dose typically providing $1464 \mathrm{mg}$ of Phosphatides. The EPA group consumed three softgel of High EPA Formula (MorEPA, Minami Nutrition, UK), with the daily dose providing 1770 $\mathrm{mg}$ EPA and $390 \mathrm{mg}$ docosahexenoic acid (DHA). The Vit-D group consumed one softgel of Vitamin D3 (Now Foods, Bloomingdale, U.S.A.), each dose providing 1,000 IU of Vitamin D3. The participants consumed the nutritional supplements during the two weeks of limb immobilisation. Participants were asked to maintain their habitual diet and not to perform any unaccustomed strenuous exercise during the 2 weeks of immobilisation and remobilisation. To monitor this, during the immobilisation period participants completed a 3-day food diary, a daily activity log (including sling-wear hours) and wore a pedometer (Omron Walking style III step counter, Omron Healthcare Co., Ltd, Kyoto, Japan) to record the number of steps taken each day. The food diaries were analysed for macronutrient and micronutrient average intake using Microdiet Plus 1.2 (Microdiet, Downlee Systems Ltd, UK). All participants recorded their daily sling wear hours. In addition, since they were university-based local recruits, they 
were in full view of at least one member of the research team and/or other university staff on campus who had been made aware of the study requirement, during their hours on campus. Last but not least, participants were questioned during their final testing session, to confirm their compliance with the protocol.

\section{Muscle and sub-cutaneous adipose thickness measures}

All images were recorded after approximately 20 minutes seated rest to avoid fluid shifts that might induce interstitial and/or intracellular changes (19). Images of the muscle and sub-cutaneous adipose tissue of the upper arm were obtained using B-mode ultrasonography (AU5, Esaote, Genoa, Italy). A 7.5-MHz linear phased-array probe (image depth: 37.1$92.8 \mathrm{~mm}$ ) was applied in the sagittal plane with minimal pressure to the tissue area of interest to avoid image distortion. This method has previously been shown to be highly reliable for determining muscle and adipose thickness (20-22). Images were recorded using Adobe Premiere 6.0 (Adobe Systems, USA) and stored for later analysis.

Images were obtained with the participant in an upright, seated position with their arm abducted square to the body (elbow at $180^{\circ}$ ) and resting on the ultrasound machine. In the upper arm, the proximal and distal insertions of the biceps and triceps brachii were identified by sonography and marked on the skin. The midpoint (L50) and a third of the distance (L33) from the distal end of the biceps and triceps brachii were identified and marked onto the skin. Upper arm ultrasonography images were collected in the sagittal plane, at both sites on both the biceps and triceps brachii. Muscle thickness was measured as the distance from the top of the superficial muscle aponeurosis to the bone at both sites along the biceps and triceps brachii. Ultrasound assessment of muscle tissue content has previously been validated (23-26). Subcutaneous adipose thickness was measured as the distance from the bottom of the epidermis to the top of the superficial muscle aponeurosis in the biceps and triceps at both sites. Each of these distances were measured at three standardised points along the width of the probe to obtain average muscle and subcutaneous adipose thicknesses using ImageJ analysis software (ImageJ 1.37, Maryland, USA).

\section{Body composition analysis}

Body composition was determined using dual-energy X-ray absorptiometry (DXA) scanner (Hologic Discovery; Vertec Scientific, Reading, Berkshire, UK). Whole body scans were performed lasting approximately seven minutes with a dosearea product (DAP) of $21 \mathrm{cGy}^{*} \mathrm{~cm}^{2}$. The appendicular mass was isolated from the trunk and head using DXA regional computer-generated default lines, with manual adjustment, on the anterior view planogram. Measures of BMD, bone mineral content (BMC), lean mass, fat mass and fat percentage are reported for the immobilised arm only.

\section{Arm girths}

Participants assumed a relaxed standing position with arms hanging by the sides and palms facing the hips. A measuring tape was used to measure upper arm girth at the mid-acromialradial and lower arm girth at a fixed point a third of the way (from the proximal end) along the length of the radiale-stylion. Measurements were repeated three times at each point and average girths were calculated.

\section{Measurement reliability}

All protocols were assessed for intra as well as inter-day reliability. This utilised five participants and entailed carrying out measurements three times on day 1 , and repeating these on day 2, approximately a week later. Within-day coefficient of variation $(\mathrm{CV})$ of $0.1 \%, 0.1 \%, 0.4 \%, 0.2 \%, 0.3 \%$ and $0.3 \%$, and between-day CVs of $0.4 \%, 0.2 \%, 0.6 \%, 0.2 \%$, $0.2 \%$ and $0.4 \%$ were yielded for upper arm girth, lower arm girth, biceps muscle thickness, triceps muscle thickness, biceps subcutaneous adipose thickness and triceps subcutaneous adipose thickness, respectively. DXA reliability is reported with a CV of $1.0 \%$.

\section{Statistical analyses}

Data were analysed using IBM SPSS v21 (IBM Inc, USA). The Shapiro-Wilk test revealed some of the data to be nonparametric (upper arm muscle and adipose thickness, upper and lower arm girths). The effect of immobilisation was examined by assessing the changes seen in the PLA group by either repeated measures ANOVA (parametric data) or a Friedman test (non-parametric data). Parametric change relative to baseline values (Pre-to-Post: (Post-Pre)/Pre; and Pre-to-Post2: (Post2-Pre)/Pre) were analysed using a repeated measures ANOVA, with post-hoc Bonferonni corrected 2-tailed t-tests to determine group difference. Non-parametric between group effects on change data were analysed using the Kruskal Wallis test, with post-hoc Mann-Whitney U tests. All data are presented as mean \pm standard deviation (SD). Statistical significance was set with alpha at $\leq 0.05$.

\section{Results}

The non-immobilised limb was also monitored throughout the immobilisation and supplementation phase. When the immobilised limb data was normalised for the non-immobilised limb data there was no effect on the outcome measures. Data for the non-immobilised limb is therefore not reported in this paper.

\section{Homogeneity of sample}

There were no significant differences in baseline characteristics between the groups (Table. 1). The groups similarly did not differ in baseline muscle size (e.g. bicep muscle thickness (L50) - PLA: $28.7 \pm 7.1 \mathrm{~mm}$; EPA: $31.1 \pm 5.4$ $\mathrm{mm}$; Vit-D: $32.1 \pm 6.5 \mathrm{~mm}$ ), arm girth (PLA: $28.8 \pm 3.0 \mathrm{~cm}$; 
EPA: $28.1 \pm 4.0 \mathrm{~cm}$; Vit-D: $29.4 \pm 3.2 \mathrm{~cm}$ ), body composition (e.g. lean mass - PLA: $1874.5 \pm 595.8$ g; EPA: $2361.0 \pm$ $1116.8 \mathrm{~g}$; Vit-D: $2531.1 \pm 832.6 \mathrm{~g})$.

\section{Table 1}

Baseline characteristics of all participants

\begin{tabular}{lccc}
\hline & PLA & EPA & Vit-D \\
\hline Age (years) & $26 \pm 6.7$ & $19 \pm 1.6$ & $23 \pm 5.9$ \\
Gender & & & \\
Males & $\mathrm{n}=2$ & $\mathrm{n}=4$ & $\mathrm{n}=3$ \\
Females & $\mathrm{n}=6$ & $\mathrm{n}=4$ & $\mathrm{n}=5$ \\
Height $(\mathrm{cm})$ & $168.7 \pm 11.1$ & $169.5 \pm 12.0$ & $172.2 \pm 8.0$ \\
Weight $(\mathrm{kg})$ & $69.1 \pm 14.2$ & $69.6 \pm 23.1$ & $75.2 \pm 14.5$ \\
BMI & $24.1 \pm 3.7$ & $24.1 \pm 5.1$ & $24.4 \pm 5.0$ \\
\hline
\end{tabular}

Mean values \pm SD for baseline characteristics.

\section{Figure 1}

Image demonstrating correct sling wear

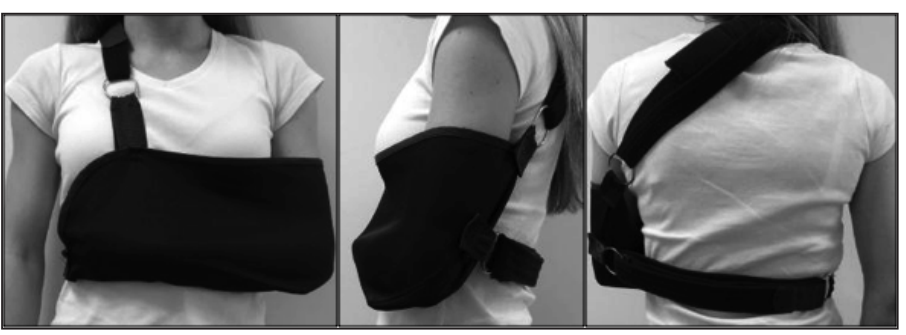

Front, side and back view demonstrating sling wear. Sling wear was initially demonstrated by the experimenter and on subsequent applications, sling application was selfadministered by each participant. The sling was worn for a minimum of nine waking hours a day for 14 days. The elbow joint was positioned at approximately a $90^{\circ}$ angle.

\section{Daily physical activity and nutritional intake}

No significant differences were observed in calorific intake (PLA: males $3134 \pm 247 \mathrm{kcal} /$ day and females $1352 \pm 506 \mathrm{kcal} /$ day; EPA: males $1826 \pm 780 \mathrm{kcal} /$ day and females $1468 \pm 121$ $\mathrm{kcal} /$ day; Vit-D: males $2658 \pm 679 \mathrm{kcal} /$ day and females 1541 $\pm 431 \mathrm{kcal} /$ day $(\mathrm{p}>0.05)$ ) or in habitual physical activity (PLA: males $8216 \pm 34$ steps/day and females $6361 \pm 1681$ steps/day; EPA: males $9648 \pm 2934$ steps/day and females $5410 \pm 2231$ steps/day; Vit-D: males $7795 \pm 1050$ steps/day and females $5156 \pm 1940$ steps/day $(\mathrm{p}>0.05))$ between the groups during the course of the immobilisation. This effect was true for the EPA, Vit-D and PLA groups. Further diet composition analyses revealed no group differences in protein (PLA: $1.1 \pm 0.3 \mathrm{~g} \cdot \mathrm{kg}$ 1·bw/day; EPA: $1.0 \pm 0.3 \mathrm{~g} \cdot \mathrm{kg}-1 \cdot \mathrm{bw} / \mathrm{day}$; Vit-D: $1.0 \pm 0.6 \mathrm{~g} \cdot \mathrm{kg}-$ $1 \cdot$ bw/day), carbohydrate (PLA: $3.1 \pm 1.5 \mathrm{~g} \cdot \mathrm{kg}-1 \cdot \mathrm{bw} /$ day; EPA: $3.1 \pm 1.1 \mathrm{~g} \cdot \mathrm{kg}-1 \cdot \mathrm{bw} /$ day; Vit-D: $3.0 \pm 1.0 \mathrm{~g} \cdot \mathrm{kg}-1 \cdot \mathrm{bw} / \mathrm{day})$, fat (PLA: $1.0 \pm 0.5 \mathrm{~g} \cdot \mathrm{kg}-1 \cdot \mathrm{bw} / \mathrm{day}$; EPA: $1.0 \pm 0.5 \mathrm{~g} \cdot \mathrm{kg}-1 \cdot \mathrm{bw} / \mathrm{day}$; Vit-D: $1.0 \pm 0.3 \mathrm{~g} \cdot \mathrm{kg}-1 \cdot \mathrm{bw} /$ day $)$, vitamin D (PLA: $1.7 \pm 0.7 \mathrm{ug} /$ day; EPA: $1.6 \pm 0.9 \mathrm{ug} /$ day; Vit-D: $2.0 \pm 1.6 \mathrm{ug} /$ day) or $\omega-3$ (PLA: $0.37 \pm 0.30 \mathrm{~g} /$ day; EPA: $0.36 \pm 0.19 \mathrm{~g} / \mathrm{day}$; Vit-D: 0.36 $\pm 0.20 \mathrm{~g} /$ day) intake over the immobilisation period between the three groups.

Results of the questionnaire revealed that participants walked to and from work, university and/or shopping a minimum of 30 minutes per day. Examination of the activity diaries completed by the participants themselves during the immobilisation period, confirmed that the majority of participants spent at least half an hour outside each day.

\section{Figure 2}

Muscle thickness changes in response to immobilisation and supplementation

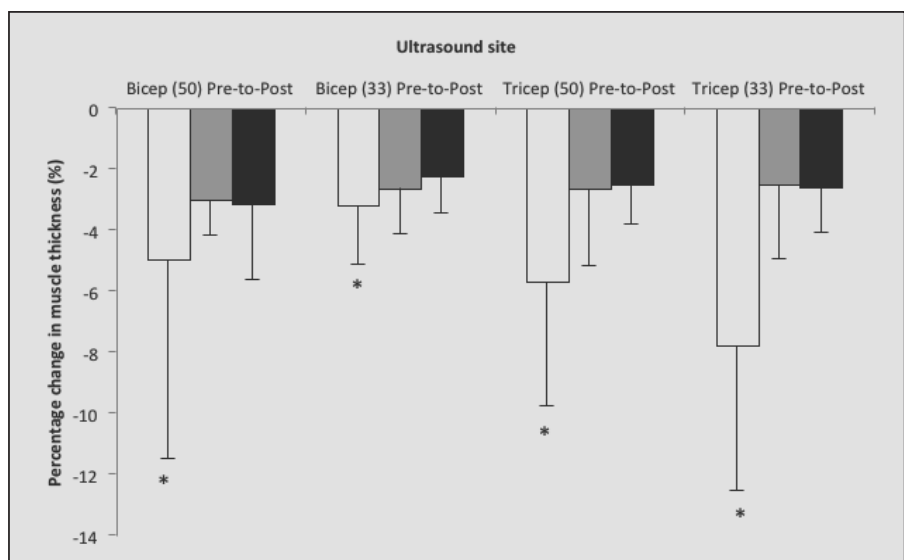

Percentage change (Mean difference $(\%) \pm$ SD) in muscle thickness from Pre-to-Post for PLA (white bars), EPA (grey bars) and Vit-D (black bars) at the midpoint (L50) and a third of the distance (L33) along the length of the biceps and triceps brachii. * Significant difference between Pre and Post immobilisation in the PLA group.

\section{Muscle and sub-cutaneous adipose thickness measures}

Muscle thickness decreased from Pre to Post at both sites (L50 and L33) on the biceps and triceps brachii $(\mathrm{p}<0.05)$ in the placebo group. The percentage decrease in muscle thickness was significantly greater in the triceps brachii (L50 \& L33) than the biceps brachii (L33) $(\mathrm{p}<0.05)$ The percentage change in muscle thickness at both sites on the biceps and triceps brachii did not significantly differ between the groups (Figure. 2), though both EPA and Vit-D groups showed a non-significant trend towards differing from the PLA group. Sub-cutaneous adipose thickness increased significantly post immobilisation at L50 of the biceps brachii $(\mathrm{p}<0.05)$, with no significant change at L33 of the biceps brachii or either site on the triceps brachii. The percentage change in adipose thickness was not significantly different between groups at both sites on the triceps brachii (Figure 3). Percentage change in adipose thickness was significantly greater in the PLA than the EPA group $(\mathrm{p}<0.05)$ and in the Vit-D than the EPA group $(\mathrm{p}<0.05)$ at L50 and L33 along the biceps brachii.

\section{Body composition}

There was a significant decrease in lean mass Post immobilisation $(\mathrm{p}<0.05)$ but no significant effect of supplement group on the percentage change in lean mass (Figure 4). Percentage changes in $\mathrm{BMC}, \mathrm{BMD}$, fat mass and fat percentage 
are displayed in Table 2. No significant change was observed in BMD, fat mass or fat percentage. There was a significant decrease in BMC from Pre to Post immobilisation in the PLA group $(\mathrm{p}<0.05)$. There was no significant difference in percentage change in $\mathrm{BMC}, \mathrm{BMD}$, fat mass or fat percentage between the groups Post or Post2 ( $>00.05)$.

\section{Figure 3}

Sub-cutaneous adipose thickness changes in response to immobilisation and supplementation

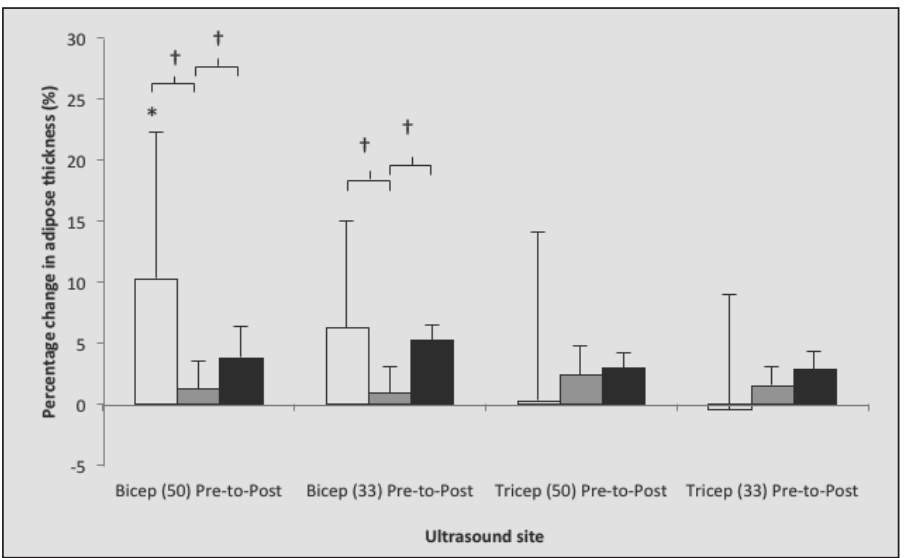

Percentage change (Mean difference $(\%) \pm \mathrm{SD}$ ) in adipose thickness from Pre-to-Post for PLA (white bars), EPA (grey bars) and Vit-D (black bars) at the midpoint (L50) and a third of the distance (L33) along the length of the biceps and triceps brachii. * Significant difference between Pre and Post immobilisation in the PLA group. $†$ Significant difference in $\%$ change between groups.

\section{Arm girths}

Upper and lower arm girths significantly decreased from Pre to Post immobilisation $(\mathrm{p}<0.05)$ in the PLA group. There was no significant difference in the percentage change in upper or lower arm girth between groups (Figure 5).

\section{Figure 4}

Lean mass changes in response to immobilisation and supplementation

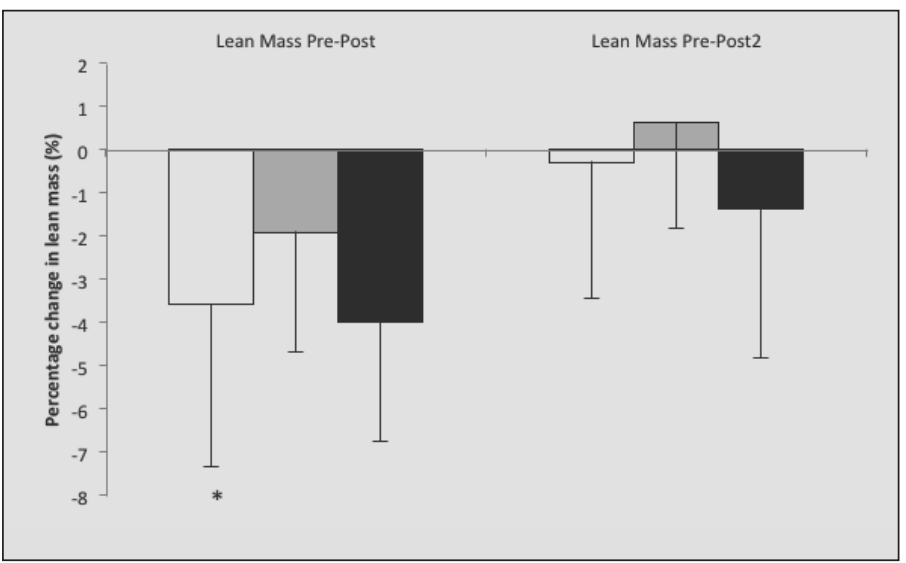

Percentage change $(\% \pm \mathrm{SD})$ in lean mass from Pre-to-Post and Pre-to-Post 2 for PLA (white bars), EPA (grey bars) and Vit-D (black bars). * Significant difference between Pre and Post immobilisation in the PLA group.
Figure 5

Arm girth changes in response to immobilisation and supplementation

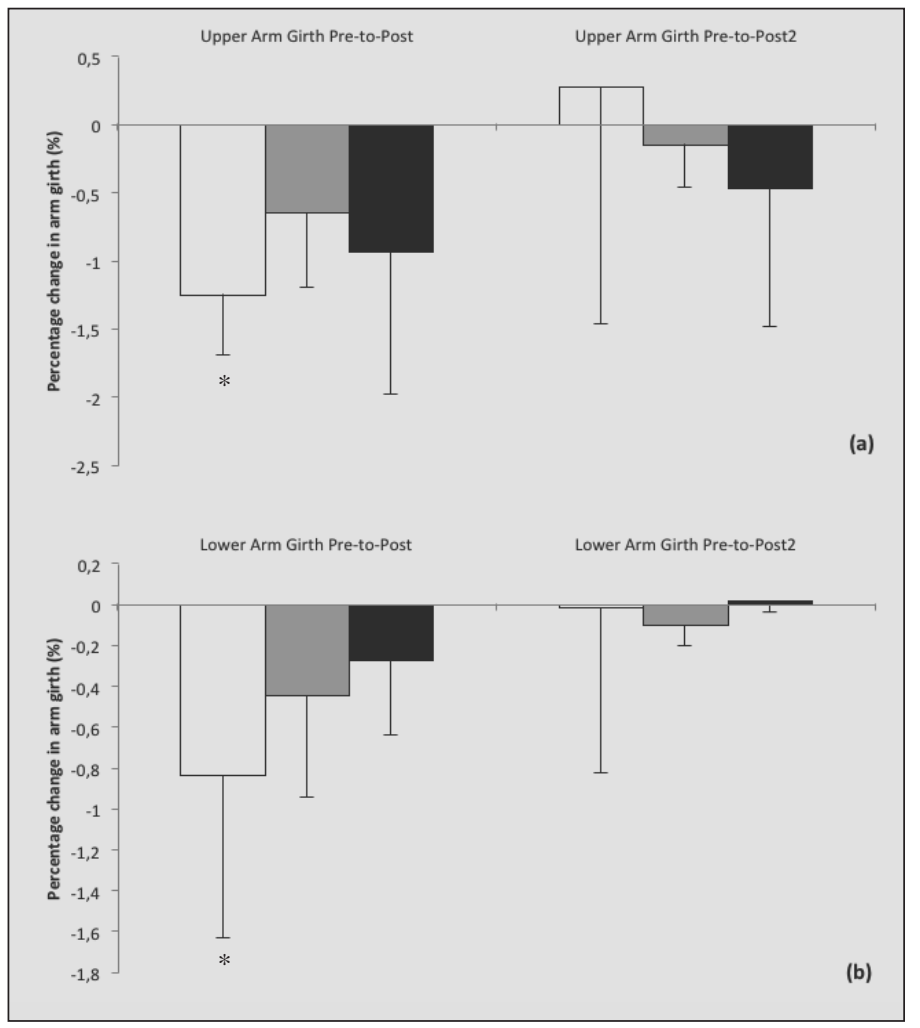

Percentage change $(\% \pm \mathrm{SD})$ in upper (a) and lower (b) arm girth from Pre-to-Post and Pre-to-Post2 for PLA (white bars), EPA (grey bars) and Vit-D (black bars). * Significant difference between Pre and Post immobilisation in the PLA group.

\section{Discussion}

The purpose of this study was to determine the role that two potential protein-sparing modulators (EPA or vitamin D supplementation) may play in attenuating the deleterious physiological changes induced through 2 weeks of 9-wakinghours-per-day combined arm and shoulder immobilisation. We hypothesised that muscle thickness, lean mass and arm girth would decrease with limb immobilisation. We found evidence to support this, with significant decreases in muscle thickness (PLA: -3.2 to $-7.8 \%$ dependent on anatomical site), arm girth (PLA: -0.8 to $-1.3 \%$ dependent on anatomical site) and lean mass (PLA: $-3.6 \%$ for the immobilised limb). In addition, we found a significant increase in sub-cutaneous adipose thickness (PLA: 10.3\%) and a significant decrease in BMC (PLA: -2.3\%). It was also hypothesised that EPA would be the most effective supplement at minimising the effects of immobilisation. In fact, our data show that neither EPA nor vitamin $\mathrm{D}$ had any significant effect on the responses to non-injurious immobilisation, other than on the associated accumulation of sub-cutaneous fatty tissue. Nonetheless, a few trends towards attenuations in deleterious physiological events were observed, in the EPA and Vit-D treated groups. 
OMEGA-3 FATTY ACIDS AND VITAMIN D IN IMMOBILISATION

Table 2

Changes in immobilised limb composition in response to immobilisation and supplementation

\begin{tabular}{|c|c|c|c|c|c|c|}
\hline \multicolumn{3}{|c|}{ PLA } & \multicolumn{2}{|l|}{ EPA } & \multicolumn{2}{|l|}{ Vit-D } \\
\hline & Pre-to-Post & Pre-to-Post 2 & Pre-to-Post & Pre-to-Post 2 & Pre-to-Post & Pre-to-Post2 \\
\hline $\mathrm{BMC}$ & $-2.3 \pm 1.5 *$ & $-1.5 \pm 1.1$ & $-0.3 \pm 1.0$ & $0.6 \pm 1.9$ & $-0.7 \pm 1.9$ & $-0.6 \pm 0.7$ \\
\hline BMD & $-1.6 \pm 2.6$ & $0.4 \pm 2.2$ & $-0.5 \pm 3.2$ & $-0.3 \pm 1.1$ & $0.0 \pm 1.5$ & $0.5 \pm 2.1$ \\
\hline Fat mass & $2.0 \pm 2.4$ & $-1.4 \pm 6.7$ & $3.2 \pm 3.2$ & $0.2 \pm 1.7$ & $2.0 \pm 3.5$ & $1.9 \pm 1.8$ \\
\hline Fat $\%$ & $2.1 \pm 5.9$ & $0.0 \pm 5.2$ & $2.6 \pm 5.3$ & $-1.2 \pm 4.3$ & $3.4 \pm 2.6$ & $0.4 \pm 3.6$ \\
\hline
\end{tabular}

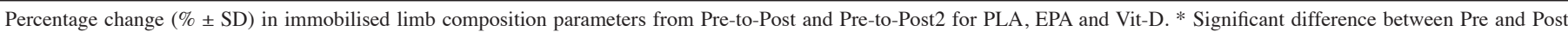
immobilisation in the PLA group.

We discuss the observed trends for the attenuation of some parameters.

A significant change in upper limb muscle thickness is evidenced in our ultrasound data, with a significant decrease in biceps and triceps brachii muscle thickness with immobilisation. The ultrasound data demonstrate a greater decrease in triceps brachii muscle (L50 and L33) thickness than the biceps brachii (L33) in the PLA group. We had, in fact, expected the muscle held in the shortened position (i.e. the biceps) to be impacted on more than the muscle held in the lengthened position (i.e. the triceps); previous research suggests that sarcomeres are added in series when the muscle is immobilised in the lengthened position, and sarcomeres are lost when the muscle is immobilised in the shortened position (27). In the present study, the elbow was immobilised at a $90^{\circ}$ angle and, as such, would not have exerted maximal lengthening or shortening on the triceps and biceps brachii. The EPA and Vit-D supplementation groups demonstrated non-significant trends towards smaller decreases in muscle thickness in response to immobilisation, suggesting that EPA and vitamin D may have some role to play in attenuating muscle atrophy associated with disuse. A recent study in an animal model demonstrated distinct effects of EPA and DHA on protein metabolism (protein synthesis and breakdown) with EPA showing a greater ability to result in skeletal muscle protein accretion. Further work in humans is required to further investigate this and the effects EPA may have in human disuse models. Indeed, it is likely that the dose, duration and/or whole body measurement of muscle mass that was adopted in these animal studies diminished the comparability of animal models from our immobilisation model.

Upper and lower arm girths were shown to decrease significantly post immobilisation in the PLA group. We used girth as a gross marker of skeletal muscle atrophy, as previously used by Matsumura et al (28). A decrease in upper arm girth (in the PLA group) suggests a decrease in muscle CSA in the upper arm and this supports our ultrasound data along with previous findings of a decrease in elbow flexor muscle CSA and volume with arm immobilisation (29). The decrease in lower arm girth suggests a decrease in forearm muscle CSA, and this is in line with finding from Miles et al. who reported a decrease in forearm muscle CSA with 9 days arm casting (30). The significant decrease in limb CSA is reflected in our DXA data, with a significant decrease in lean mass with immobilisation. The decrease in upper arm girth appeared to be attenuated by EPA and vitamin D supplementation, with EPA having the greater effect. Similarly, in the lower arm, supplementation showed a trend towards attenuating the losses in arm girth, this time with vitamin D having a slightly greater effect. The EPA group appear to show a smaller decrease in lean mass than both other groups.

The ultrasound data revealed no significant change in subcutaneous adipose thickness in the triceps brachii over the immobilisation period. Biceps sub-cutaneous adipose thickness, however, did significantly increase at the midpoint site in the placebo group. Manini et al. (6) reported a significant increase in intermuscular adipose tissue and no significant change in subcutaneous adipose tissue in response to 4-weeks unilateral lower limb suspension. The difference between the response of sub-cutaneous adipose tissue to disuse in the current study in comparison to Manini et al. may be due to the different techniques used to assess the parameter (i.e. ultrasound vs. magnetic resonance imaging) and/or the differing modes of immobilisation (i.e. arm immobilisation vs. unilateral lower limb suspension). It is possible that the more stringent immobilisation in their study (throughout daily mobile activities, for four weeks), as well as the adiposity site (intermuscular), may account for the fact that they observed significant increases in adiposity. The present study partially agrees with their findings as our data showed a significant increase at one region, subcutaneously, along the upper arm (i.e. 50\% of upper limb length). The EPA group demonstrated a significantly smaller increase in biceps sub-cutaneous adipose thickness following immobilisation than both the PLA and Vit-D groups. This effect may, indeed, be meaningful given that diet was monitored and the dietary records demonstrated neither time nor between group differences in total calories, macronutrients or vitamin D intakes, throughout the study. EPA supplementation potential for attenuating increases in subcutaneous adipose thickness in response to disuse. The Vit-D group also demonstrated a trend towards an attenuation in the increase of sub-cutaneous adipose thickness in comparison to 
the PLA group.

DXA analysis revealed no significant changes in fat mass or fat percentage with limb immobilisation. The difference in the response of adiposity to disuse in the current study in comparison to Manini et al. (6), who reported a significant increase in intermuscular adipose tissue, may be due to the different techniques used to assess the parameter (i.e. DXA vs. magnetic resonance imaging) or the different mode and/ or duration of disuse (i.e. 2 weeks arm immobilisation vs. 4 weeks unilateral lower limb suspension). Interestingly, the increases we reported in sub-cutaneous adipose thickness were not matched by our DXA analysis of fat mass or percentage. Possible explanations for this include: a) the DXA takes the average of the whole limb rather than regional data and hence would have averaged-out the regional changes observed with the ultrasound; b) it is possible that the tissue density changes with immobilisation mean that the DXA may have missqualified muscle and fat after immobilisation (31).

We reported no significant change in BMD $(-0.8 \% /$ week $)$, but a significant decrease in BMC (-1.2\%/week) following immobilisation. Decreases in bone are often reported in more severe and longer periods of disuse. For example, Rittweger et al. reported significant decreases in BMC of the tibia ( -0.1 to $-0.5 /$ week) and radius ( -0.03 to $-0.05 \% /$ week) in response to 90 days bed rest (5). Marchetti et al. found significant decreases in BMD (-1.0 to 2.3\%/week) in response to 6 weeks arm immobilisation, however, the participants had also undergone surgery, which could contribute to greater decreases in BMD (32). Values for BMC and BMD changes with immobilisation showed a trend towards attenuation of changes with EPA and vitamin D supplementation. Previous research indicates that BMD is affected by changes in body weight and composition (33), therefore our observed changes in muscle and subcutaneous adipose thickness may have influenced our BMD values. The value of BMD can also be limited by the inherent limitations of using a two-dimensional $\mathrm{x}$-ray projection to estimate bone area and geometrical changes. The use of peripheral quantitative tomography (pQCT) may have been more advantageous to examine these specific changes.

A recent study in our own laboratory, found that a lower dose of EPA and DHA than that used in the current study, was not sufficient to decrease inflammation (34). We therefore chose to increase the EPA and DHA of our $\omega-3$ supplement in the current study, in line with previous levels used in the literature (35-37). The vitamin D dose of $1000 \mathrm{IU}$ is equal to 25 ug. Public Health England suggest that adults at risk of vitamin D deficiency should take a daily supplement containing 10 ug of vitamin D (38). Our selected dose is much higher than this and as such should be sufficient in the healthy population used in the current study. The formula of each supplement was chosen so that the dose provided would be able to be bought over the counter and be taken without prescription. The lack of the effectiveness of vitamin D and EPA in the current study may be due to the dosage of the supplements used and therefore this needs to be altered, potentially increased, in future studies.

The NHS consensus on vitamin D states that if people achieve a sufficient supply of vitamin D in the summer, most should keep levels greater than the deficiency threshold of $25 \mathrm{nmol} / 1$ in winter even without supplements (39). In the current study, participants reported that they typical spent at least 30 minutes outdoors every day of the week and as such would reach the necessary sunlight exposure for adequate vitamin D levels. Research has shown that light of even relatively low intensity ( $\sim 180$ lux) significantly phase-shifts the human circadian rhythm [40]. We therefore suggest that the current population are unlikely to be vitamin $\mathrm{D}$ deficient. The 'healthy, presumably non-deficient' status of the participants may have been another reason for the lack of effectiveness of the supplementation in the current study. In a recent study, it was reported that in an elderly population, most participants were unable to meet the recommended daily omega-3 ( $\omega-3)$ intake without the use of fish oil supplements (41). Future studies need to recruit those lacking in physiological reserve and those more at risk of malnutrition/deficiencies e.g. older and/or injured persons. Future studies also need to assess endocrine markers, including serum EPA \& DHA, 25(OH) D3 levels (biomarker for vitamin D status) and parathyroid hormone (PTH) to observe the effects of supplementation.

Blinding of $\omega-3$ supplementation is sometimes an issue in studies due to the fact that it often repeats on participants. On completion of the study, participants were asked whether they were able to identify what supplement they believed they were taking. None of the participants correctly guessed the supplement they were taking and in fact, two participants receiving the placebo supplement believed they were taking an $\omega-3$ supplement.

\section{Conclusion}

In accordance with previous research, we demonstrated a decrease in muscle thickness, arm girth and lean mass with short-term upper limb immobilisation. We also observed a significant decrease in BMC, with no observed effect of immobilisation on fat mass or BMD. At the current dosage, neither vitamin D nor EPA supplementation impacted on these parameters at a statistically significant level. Interestingly nonetheless, we have observed that in the case of sub-cutaneous adiposity on the biceps brachii, there is a protective effect of EPA supplementation against limb immobilisation. We propose that the model used in the current study could have relevance to a sporting population in which short-term immobilisation may be prescribed (e.g. treatment for minor injury) or in clinical populations (e.g. injury/surgery induced short-term immobilisation/bed-rest). A short-term model such as this is relatively less invasive and inconvenient for participants and therefore provides a model to be used to assess other supplements and treatments in future studies.

Ethics declaration: All experimental procedures were conducted in accordance with 


\section{OMEGA-3 FATTY ACIDS AND VITAMIN D IN IMMOBILISATION}

the guidelines in the Declaration of Helsinki and approved by the Ethics Committee of Manchester Metropolitan University.

Acknowledgements: The authors thank HEAL (Health, Exercise and Active Living Research Centre) for funding the study. We would also like to thank the participants for their time and persistence with the study.

Conflict of Interest Disclosures: The authors do not have any conflicts of interest.

\section{References}

1. de Boer MD, Maganaris CN, Seynnes OR, Rennie MJ, Narici MV. Time course of muscular, neural and tendinous adaptations to 23 day unilateral lower-limb suspension in young men. J Physiol, 2007;583:1079-91.

2. LeBlanc AD, Schneider VS, Evans HJ, Pientok C, Rowe R, Spector E. Regional changes in muscle mass following 17 weeks of bed rest. J Appl Physiol, 1992;73:2172-8.

3. Veldhuizen JW, Verstappen FT, Vroemen JP, Kuipers H, Greep JM. Functional and morphological adaptations following four weeks of knee immobilization. Int J Sports Med, 1993;14:283-7.

4. Bloomfield SA. Changes in musculoskeletal structure and function with prolonged bed rest. Med Sci Sports Exerc, 1997;29:197-206.

5. Rittweger J, Frost HM, Schiessl H, Ohshima H, Alkner B, Tesch P, Felsenberg D. Muscle atrophy and bone loss after 90 days' bed rest and the effects of flywheel resistive exercise and pamidronate: results from the LTBR study. Bone, 2005;36:1019-29.

6. Manini TM, Clark BC, Nalls MA, Goodpaster BH, Ploutz-Snyder LL, Harris TB. Reduced physical activity increases intermuscular adipose tissue in healthy young adults. Am J Clin Nutr, 2007;85:377-84.

7. National Joint Registry. National Joint Registry for England and Wales 9th Annual Report. 2012; Available from: http://www.njrcentre.org.uk/njrcentre/Portals/0/ Documents/England/Reports/9th_annual_report/NJR\%209th\%20Annual\% 20 Report\%202012.pdf.

8. Moylan KC, Binder EF. Falls in older adults: risk assessment, management and prevention. Am J Med, 2007;120:493 e1-6.

9. A Report of the Panel on Macronutrients, Subcommittees on Upper Reference Levels of Nutrients and Interpretation and Uses of Dietary Reference Intakes, the Standing Committee on the Scientific Evaluation of Dietary Reference Intakes. Dietary Fats: Total Fat and Fatty Acids, in Dietary Reference Intakes for Energy, Carbohydrate, Fiber, Fat, Fatty Acids, Cholesterol, Protein, and Amino Acids (Macronutrients), A Report of the Panel on Macronutrients, Subcommittees on Upper Reference Levels of Nutrients and Interpretation and Uses of Dietary Reference Intakes, and the Standing Committee on the Scientific Evaluation of Dietary Reference Intakes, Editors., The National Academies Press: Washington, DC.2005;422-541.

10. Magee P, Pearson S, Allen J. The omega-3 fatty acid, eicosapentaenoic acid (EPA), prevents the damaging effects of tumour necrosis factor (TNF)-alpha during murine skeletal muscle cell differentiation. Lipids Health Dis, 2008;7:24.

11. Grosset JF, Onambele-Pearson G. Effect of foot and ankle immobilization on leg and thigh muscles' volume and morphology: a case study using magnetic resonance imaging. Anat Rec (Hoboken), 2008;291:1673-83.

12. de Boer MD, Selby A, Atherton P, Smith K, Seynnes OR, Maganaris CN, Maffulli N, Movin T, Narici MV, Rennie MJ. The temporal responses of protein synthesis, gene expression and cell signalling in human quadriceps muscle and patellar tendon to disuse. J Physiol, 2007;585:241-51.

13. Ferrando AA, Lane HW, Stuart CA, Davis-Street J, Wolfe RR. Prolonged bed rest decreases skeletal muscle and whole body protein synthesis. Am J Physiol, 1996;270:E627-33.

14. Ross CA, Taylor CL, Yaktine AL, Del Valle HB. Dietary Reference Intakes for Adequacy: Calcium and Vitamin D, in Dietary Reference Intakes for Calcium and Vitamin D, Ross CA, et al., Editors., The National Academies Press: Washington, DC. 2011;345-402.

15. Ceglia L, Harris SS. Vitamin D and Its Role in Skeletal Muscle. Calcif Tissue Int, 2012;92:151-162.

16. Grundberg E, Brandstrom H, Ribom EL, Ljunggren O, Mallmin H, Kindmark A. Genetic variation in the human vitamin D receptor is associated with muscle strength, fat mass and body weight in Swedish women. Eur J Endocrinol, 2004;150:323-8.

17. Stewart CE, Rittweger J. Adaptive processes in skeletal muscle: molecular regulators and genetic influences. J Musculoskelet Neuronal Interact, 2006;6:73-86.

18. Bischoff HA, Stahelin HB, Dick W, Akos R, Knecht M, Salis C, Nebiker M, Theiler R, Pfeifer M, Begerow B, Lew RA, Conzelmann M. Effects of vitamin D and calcium supplementation on falls: a randomized controlled trial. J Bone Miner Res, 2003;18:343-51.

19. Berg HE, Tedner B, Tesch PA. Changes in lower limb muscle cross-sectional area and tissue fluid volume after transition from standing to supine. Acta Physiol Scand, 1993;148:379-85.

20. Miyatani M, Kanehisa H, Ito M, Kawakami Y, Fukunaga T. The accuracy of volume estimates using ultrasound muscle thickness measurements in different muscle groups. Eur J Appl Physiol, 2004;91:264-72.

21. Miyatani M, Kanehisa H, Kuno S, Nishijima T, Fukunaga T. Validity of ultrasonograph muscle thickness measurements for estimating muscle volume of knee extensors in humans. Eur J Appl Physiol, 2002;86:203-8.

22. Onambele GL, Narici MV, Maganaris CN. Calf muscle-tendon properties and postural balance in old age. J Appl Physiol, 2006;100:2048-56.

23. Abe T, Loenneke JP, Thiebaud RS. Ultrasound assessment of hamstring muscle size using posterior thigh muscle thickness. Clinical Physiology and Functional Imaging: 2014; doi: $10.1111 / \mathrm{cpf} .12214$.

24. Dupont AC, Sauerbrei EE, Fenton PV, Shragge PC, Loeb GE, Richmond FJ. Realtime sonography to estimate muscle thickness: comparison with MRI and CT. Journal of clinical ultrasound : JCU, 2001;29:230-6.

25. Giles LS, Webster KE, McClelland JA, Cook J. Can ultrasound measurements of muscle thickness be used to measure the size of individual quadriceps muscles in people with patellofemoral pain? Physical Therapy in Sport, 2014;16:45-52.

26. Temes WC, Temes Clifton A, Hilton V, Girard L, Strait N, Karduna A. Reliability and validity of thickness measurements of the supraspinatus muscle of the shoulder: an ultrasonography study. Journal of sport rehabilitation, Technical Notes, 2014.

27. Williams PE, Goldspink G. The effect of immobilization on the longitudinal growth of striated muscle fibres. J Anat, 1973;116:45-55.

28. Matsumura M, Ueda C, Shiroishi K, Esaki K, Ohmori F, Yamaguchi K, Ichimura S, Kurosawa Y, Kime R, Osada T, Murase N, Katsumura T, Hoshika A, Hamaoka $\mathrm{T}$. Low-volume muscular endurance and strength training during 3-week forearm immobilization was effective in preventing functional deterioration. Dyn Med, 2008;7:1-8.

29. Yue GH, Bilodeau M, Hardy PA, Enoka RM. Task-dependent effect of limb immobilization on the fatigability of the elbow flexor muscles in humans. Exp Physiol, 1997;82:567-92.

30. Miles MP, Clarkson PM, Bean M, Ambach K, Mulroy J, Vincent K. Muscle function at the wrist following $9 \mathrm{~d}$ of immobilization and suspension. Med Sci Sports Exerc, 1994;26:615-23.

31. Roubenoff R, Kehayias JJ, Dawson-Hughes B, Heymsfield SB. Use of dual-energy $\mathrm{X}$-ray absorptiometry in body-composition studies: not yet a «gold standard». Am J Clin Nutr, 1993;58:589-91.

32. Marchetti ME, Houde JP, Steinberg GG, Crane GK, Goss TP, Baran DT. Humeral bone density losses after shoulder surgery and immobilization. J Shoulder Elbow Surg, 1996;5:471-6.

33. Van Loan MD, Johnson HL, Barbieri TF. Effect of weight loss on bone mineral content and bone mineral density in obese women. Am J Clin Nutr, 1998;67:734-8.

34. Houghton D, Onambele GL. Can a standard dose of eicosapentaenoic acid (EPA) supplementation reduce the symptoms of delayed onset of muscle soreness? J Int Soc Sports Nutr, 2012;9:2.

35. Bloomer RJ, Larson DE, Fisher-Wellman KH, Galpin AJ, Schilling BK. Effect of eicosapentaenoic and docosahexaenoic acid on resting and exercise-induced inflammatory and oxidative stress biomarkers: a randomized, placebo controlled, cross-over study. Lipids Health Dis, 2009;8:36.

36. Lenn J, Uhl T, Mattacola C, Boissonneault G, Yates J, Ibrahim W, Bruckner G. The effects of fish oil and isoflavones on delayed onset muscle soreness. Med Sci Sports Exerc, 2002;34:1605-13.

37. Phillips T, Childs AC, Dreon DM, Phinney S, Leeuwenburgh C. A dietary supplement attenuates IL-6 and CRP after eccentric exercise in untrained males. Med Sci Sports Exerc, 2003;35:2032-7.

38. Public Health England. Vitamin D: All you need to know. 2014; Available from: https://www.gov.uk/government/uploads/system/uploads/attachment_data/ file/390394/A5_Vitamin_leaflet_public_FINAL_22_12_14.pdf.

39. NHS Livewell. Consensus Vitamin D position statement. 2010; Available from: http://www.nhs.uk/livewell/summerhealth/documents/concensus_statement $\% 20$ vitd_dec_2010.pdf.

40. Boivin DB, Duffy JF, Kronauer RE, Czeisler CA. Dose-response relationships for resetting of human circadian clock by light. Nature, 1996;379:540-542.

41. Pittaway JK, Chuang LT, Ahuja KD, Beckett JM, Glew RH, Ball MJ. Omega3 dietary Fatty Acid status of healthy older adults in Tasmania, Australia: an observational study. J Nutr Health Aging, 2015;19:505-10. 